\title{
Improving the Energy Efficiency of Autonomous Underwater Vehicles by Learning to Model Disturbances
}

\author{
Petar Kormushev and Darwin G. Caldwell
}

\begin{abstract}
Energy efficiency is one of the main challenges for long-term autonomy of AUVs (Autonomous Underwater Vehicles). We propose a novel approach for improving the energy efficiency of AUV controllers based on the ability to learn which external disturbances can safely be ignored. The proposed learning approach uses adaptive oscillators that are able to learn online the frequency, amplitude and phase of zeromean periodic external disturbances. Such disturbances occur naturally in open water due to waves, currents, and gravity, but also can be caused by the dynamics and hydrodynamics of the AUV itself. We formulate the theoretical basis of the approach, and demonstrate its abilities on a number of input signals. Further experimental evaluation is conducted using a dynamic model of the Girona $500 \mathrm{AUV}$ in simulation on two important underwater scenarios: hovering and trajectory tracking. The proposed approach shows significant energy-saving capabilities while at the same time maintaining high controller gains. The approach is generic and applicable not only for AUV control, but also for other type of control where periodic disturbances exist and could be accounted for by the controller.
\end{abstract}

\section{INTRODUCTION}

Autonomous Underwater Vehicles (AUVs) have existed for a long time. However, even nowadays, most of the work in the field done by offshore construction and oil companies is still being conducted by Remotely Operated Vehicles (ROVs) controlled by human operators and powered from the surface via an umbilical.

The reason for the underuse of AUVs is their limited autonomy at many levels: limited energy autonomy, limited cognitive capacity, limited adaptability to changes, limited mission re-planning ability, etc. Improving the level of autonomy of AUVs in all these different aspects is crucial for increasing their utility. The ultimate goal would be to have AUVs working fully autonomously over extended periods of time and in challenging underwater missions, which is also the main goal of the European project PANDORA [1].

Among the challenges, the energy autonomy is one of the most difficult to solve, especially since any increase in the AUV's higher-level abilities usually leads to higher computational demand and therefore even higher energy consumption.

In addition to the compulsory navigation and trajectory following tasks, there is an ever-increasing demand for complex task to be executed by AUVs. Examples include autonomous inspection of sub-sea structures in an unknown

P. Kormushev and D. G. Caldwell are with the Department of Advanced Robotics, Istituto Italiano di Tecnologia, via Morego 30, 16163 Genova, Italy. \{petar.kormushev, darwin.caldwell\} at iit.it

This work is supported by the European project PANDORA: Persistent Autonomy through learNing, aDaptation, Observation and ReplAnning, contract FP7-ICT-288273. http://persistentautonomy.com

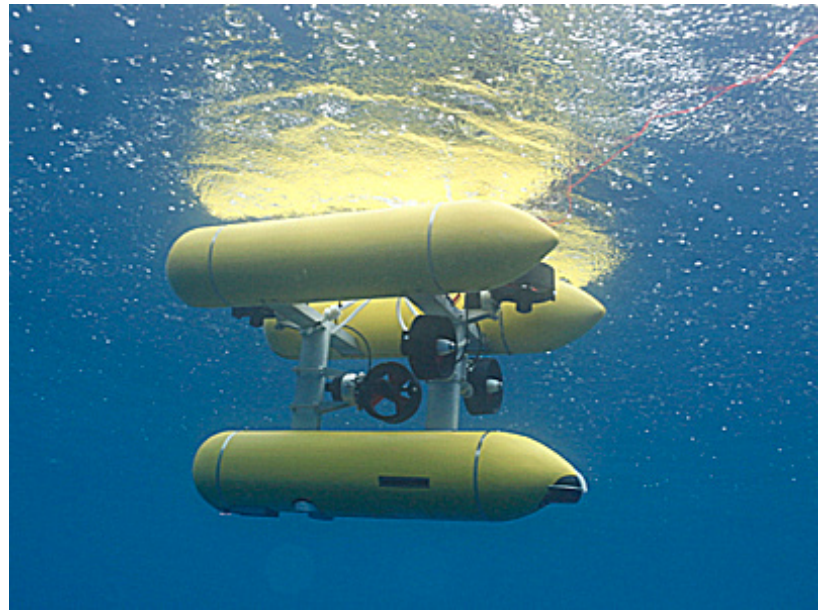

Fig. 1. The Girona 500 hover-capable AUV getting disturbed by waves at shallow depth in open water. A dynamic model of this AUV, obtained through system identification, is used for the simulated experiments described in this paper. [Photo credit: CIRS Underwater Robotics Research Center, University of Girona, Spain]

area, autonomous image mosaicing using vision and sonar, or even more demanding object manipulation tasks, such as autonomous valve turning [2].

However, unlike high-level cognitive, planning, or mapping processes, which could be switched off temporarily to conserve power, the low-level AUV controller can never be turned off. This is what makes the task of improving the energy efficiency of the AUV controller so important.

In this paper, we propose a novel approach for improving the energy efficiency of the AUV controller based on the ability to learn which external disturbances can safely be ignored. The proposed learning approach is based on the theory of synchronization and uses adaptive oscillators to learn online the frequency, amplitude and phase of periodic external disturbances. Such disturbances occur naturally in open water due to the waves, currents, and gravity, but also can be caused by the dynamics and hydrodynamics of the AUV itself. After an overview of the related work, we formulate the theoretical basis of the approach, and demonstrate its abilities on a number of input signals. In the experimental section, the approach is tested on hovering and trajectory tracking tasks, using a dynamics model of the Girona 500 AUV (shown in Fig. 1).

\section{RELATED WORK}

Most of the existing work dedicated to energy efficiency of AUVs focuses on the design of new propelling mechanisms. 
Two prominent examples are: underwater gliding and energy harvesting.

Underwater gliders are a relatively new type of AUVs that glide by controlling their buoyancy and attitude using internal actuators [3]. The reason for their energy efficiency is that they use gravity to do most of the work on propelling the vehicle. The energy required to change the buoyancy is much smaller than the energy required to propel the AUV, which makes the approach feasible. However, gliders usually have limited maneuverability and speed, which makes them impractical for intervention missions.

Energy harvesting is a promising new approach which allows an Unmanned Maritime Vehicle (UMV) to harness ocean wave energy for platform propulsion. A representative example is Liquid Robotics Wave Glider [4] which consists of two parts: a surface boat, and an underwater body with fins. The Wave Glider uses the motion of surface waves to paddle the underwater fins up and down while changing their orientation accordingly. The reason for its energy efficiency is that it harnesses the vast power of waves while needing only very little power to change the fins' orientation.

This paper addresses the problem of energy efficiency from another perspective: how to improve the energy efficiency of already existing AUVs by improving the design of their controllers. The proposed solution is based on the theory of synchronization [5], and more specifically on the so-called adaptive oscillators. The related work for them is given in Section IV after the introduction of the proposed theoretic concept for improving the energy efficiency in Section III. To the best of our knowledge, this is the first time adaptive oscillators are being applied in the domain of marine robotics. Until now, their primary field of application has been to legged robots or walking assistance devices [6][7].

\section{THEORETIC CONCEPT FOR IMPROVING THE ENERGY EFFICIENCY}

Let us consider some external disturbance applied to the AUV. Throughout the paper, we assume that this disturbance is a variable force $F_{\text {ext }}(t)$ acting on the center of mass (CoM) of the AUV. However, the same approach can easily be applied also to other types of disturbances, e.g. a torque perturbing the orientation of the AUV.

\section{A. Zero-mean periodic disturbances}

We define an external disturbance $G(t)$ to be a zero-mean periodic disturbance if the following holds true:

$$
\exists T>0 \quad \forall t_{0} \int_{t_{0}}^{t_{0}+T} G(t) d t=0,
$$

considering only non-trivial solutions (i.e. $G(t) \not \equiv 0$ ), and limiting the period $T$ to a specific range $T \in\left[T_{0}, T_{1}\right]$.

The interesting property of such zero-mean periodic disturbances is that their net effect on the state of the AUV over longer periods of time $(\gg T)$ is negligible. Thus, they could potentially be ignored by the AUV controller without affecting the long-term macro-scale tracking precision. This is where the theoretic potential for energy saving is found - by ignoring certain disturbances, instead of trying to actively counteract them, the AUV controller could save energy at the micro-scale level without compromising the overall macroscale performance.

Typical examples for such zero-mean periodic disturbances are the sea waves. Their effect can easily go as deep as tens of meters underwater. Another example is gravity, causing pendulum-like oscillations to AUVs with low CoM and positive buoyancy of the upper part (which is commonly used, to prevent excessive roll or pitch of the AUV). Yet another example is hydrodynamic oscillation at higher speed due to turbulent water flow around the AUV. Most of these disturbances could potentially be ignored (either completely or partially) by the controller in order to save energy.

However, finding such zero-mean periodic disturbances hidden within the noisy total external disturbance $F_{\text {ext }}(t)$ is not a trivial task ${ }^{1}$. Moreover, the spectrum of real-world disturbances is non-stationary, i.e. it evolves over time. Even if the spectrum was stationary, the perceived disturbance by the AUV would still vary in time due to the Doppler effect caused by the motion of the AUV itself.

In this paper, we propose a theoretic framework and show experimental results how the challenging task of identifying and tracking zero-mean periodic disturbances could be solved.

\section{B. Problem formulation}

The instantaneous total external disturbance $F_{\text {ext }}(t)$ can be represented as:

$$
F_{e x t}(t)=\sum_{i=1}^{N} G_{i}(t)+H(t)+c,
$$

where $G_{i}(t)$ are zero-mean periodic functions, $H(t)$ is a nonperiodic function which can include also random noise, and $c$ is a scalar offset. Over time, however, the functions are changing. We assume that these changes happen smoothly, without discontinuities - a reasonable assumption for physical systems without hard contact, such as free-floating AUVs.

Following this formulation, the problem is to identify as many $G_{i}$ components as possible and as accurately as possible, while simultaneously tracking their evolution over time. In order to make this hard problem more tractable, we restrict the class of zero-mean periodic functions to harmonic oscillations, i.e. having the following form:

$$
G_{i}(t)=\alpha_{i} \cos \left(w_{i} t+\phi_{i}\right)
$$

This is not a significant restriction, since harmonic oscillations are by far the most widespread waveform that occurs in nature and satisfies (1). Moreover, it is wellknown from harmonic analysis that any periodic function can be approximated arbitrarily close by a sum of harmonic oscillations.

\footnotetext{
${ }^{1} \mathrm{~A}$ simple Fourier analysis is not enough to identify reliably and track smoothly the non-stationary spectrum of the zero-mean periodic disturbances, due to artifacts caused by the sliding window and signal enveloping.
} 
Finally, the goal is to identify and track continuously over time the amplitudes $\alpha_{i}$, frequencies $w_{i}$ and phases $\phi_{i}$ of as many harmonic $G_{i}$ components of $F_{\text {ext }}$ as possible.

\section{THE ROLE OF SYNCHRONIZATION}

A conventional signal processing approach towards solving the problem from the previous section would be to apply Fourier transform (e.g. FFT) on $F_{\text {ext }}(t)$. Using a fixed-size window and an appropriate signal envelope, it would be possible to perform frequency analysis of the windowed signal. Then, by sliding the window along the signal and repeating the process at regular time instants, a spectrogram would be obtained, indicating the frequency spectrum evolution over time. This conventional paradigm, however, has a number of important drawbacks:

- It assumes stationary spectrum inside each window, which is not realistic especially when using longer windows, in order to detect lower frequencies;

- It is computationally expensive, which would diminish any potential energy savings from using it on the AUV;

- It is not dynamically consistent, because individual static frequency snapshots are being stitched together;

- It cannot be easily used to predict the future signal.

Instead, we propose a completely different paradigm for solving the problem, based on the theory of synchronization [5]. The idea is to create individual oscillators - one for each $G_{i}$ component that needs to be tracked - and synchronize them gradually with the input signal. Then, taking advantage of the dynamic consistency of the oscillators, they maintain synchrony with the harmonic components $G_{i}$ thus providing smooth output (and accurate predictions) to be used by the AUV controller. This approach has numerous advantages, which are summarized in Section VII.

In the remainder of this section, we introduce the details of the proposed synchronization approach. It is mainly based on the work of Righetti [8][9][10], Buchli [11][6], and Ijspeert. However, instead of Cartesian coordinates we use representations in polar coordinates, due to some advantages as explained in [12].

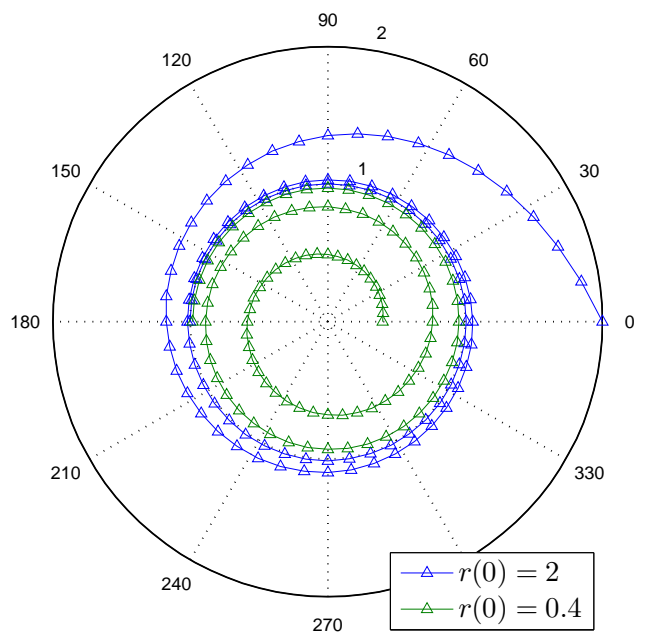

Fig. 2. Polar plot showing the convergence of two Hopf oscillators with different initial conditions towards a common attracting limit cycle $r=1$.

\section{A. The Hopf oscillator}

The simple Hopf oscillator in polar coordinates is defined by the following differential equations:

$$
\left\{\begin{array}{l}
\dot{r}(t)=\gamma\left(\mu-r(t)^{2}\right) r(t) \\
\dot{\phi}(t)=w,
\end{array}\right.
$$

where $r(t)$ is the radius, $\phi(t)$ is the phase of the twodimensional output of the system at time $t[\mathrm{~s}]$. The output of the oscillator is defined as $G(t)=r(t) \cdot \cos (\phi(t))$.

The constant $\gamma$ defines the strength of the attracting limit cycle, i.e. how fast the oscillator returns to the limit cycle after a perturbation. The oscillator has a stable limit cycle with radius $\sqrt{\mu}$. The frequency of the oscillation is defined by $w$ in units of $[\mathrm{Hz} \cdot 2 \pi]$.

The Hopf oscillator has the nice property to forget perturbations acting on $r$ after a while, as shown in Fig. 2. This is due to the limit cycle being an attractor acting against the perturbation. On the other hand, perturbations on the phase $\phi$ are remembered indefinitely [8].

\section{B. Adaptive Hopf Oscillator}

Despite its stable limit cycle, the simple Hopf oscillator cannot be applied directly for solving our problem, because it is not able to adapt its frequency nor amplitude. Instead, we propose to use an extended version of it called Adaptive Hopf Oscillator (AHO). It is based on the concept of dynamic Hebbian learning in adaptive frequency oscillators as described in the work of Righetti et al. [9].

Hebbian learning is similar to correlation-based learning and is observed in biological neural networks. It gives the AHO the ability to dynamically adapt both its frequency and amplitude to any periodic signal. The AHO embeds the learning process directly into the dynamics of the oscillator itself.

The equations governing the dynamics of the adaptive oscillator are as follows:

$$
\left\{\begin{array}{l}
\dot{r}(t)=\gamma\left(\mu-r(t)^{2}\right) r(t)+\epsilon F(t) \cdot \cos (\phi(t)) \\
\dot{\phi}(t)=w(t)-\frac{\epsilon}{r(t)} F(t) \cdot \sin (\phi(t)) \\
\dot{w}(t)=-\epsilon F(t) \cdot \sin (\phi(t)) \\
\dot{\alpha}(t)=\eta F(t) \cdot \cos (\phi(t)) \cdot r(t),
\end{array}\right.
$$

where $F(t)$ is the input (driving) signal and $\alpha(t)$ is the amplitude of the oscillation. The constants $\epsilon$ and $\eta$ act as a learning rate for the frequency and the amplitude respectively. The output of the system is redefined as $G(t)=$ $\alpha(t) \cdot r(t) \cdot \cos (\phi(t))$.

The learning rule for $\alpha$ is also based on Hebbian learning. The value of $\alpha$ increases if $F$ correlates with the output $r \cdot \cos (\phi)$ of the system, while $\eta \in(0,1)$ acts as a learning rate.

A formal proof of convergence can be found in [11]. In the case of stationary input, the adaptive oscillator is able to quickly adjust its frequency and amplitude to match the input signal, as demonstrated in Fig. 3. The speed of adaptation can be adjusted by changing the learning rates $\epsilon$ and $\eta$. 

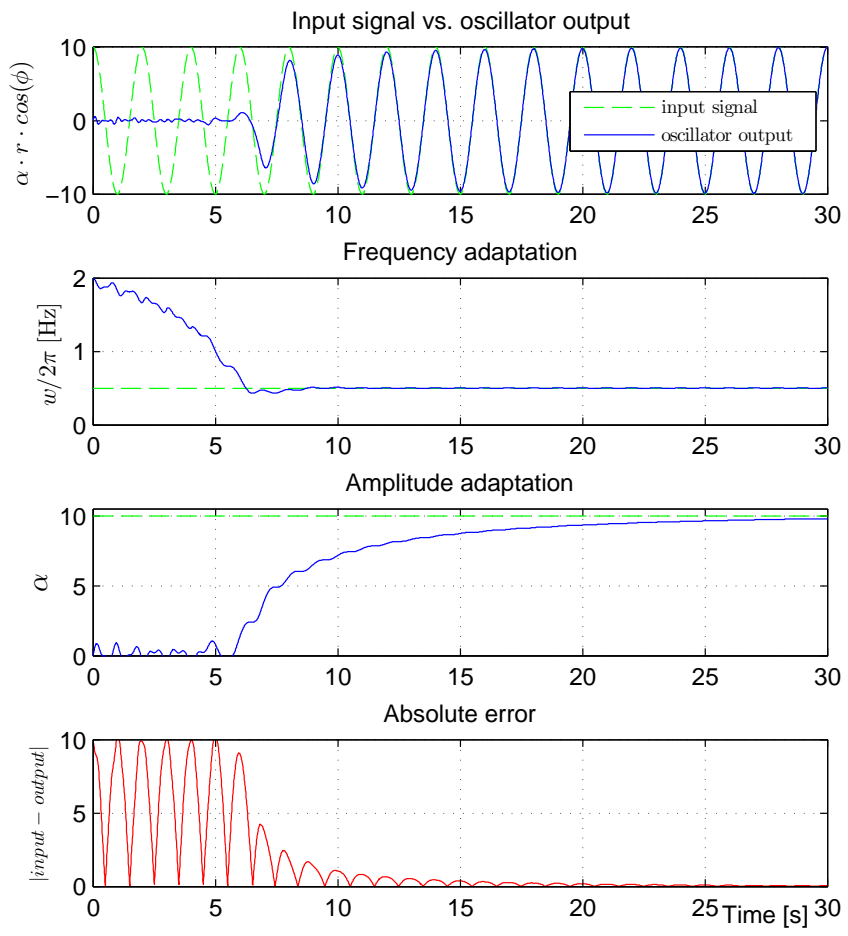

Fig. 3. A single adaptive oscillator synchronizing with an input signal.

In the case of non-stationary input, the adaptive oscillator is able to smoothly track the changes of the input frequency and amplitude, as demonstrated in Fig. 4. This tracking ability is very important since the perceived external disturbance by the AUV is influenced by its self-motion (e.g. Doppler shift).

\section{Dynamical frequency analysis}

As explained in [11], it is possible to construct a system capable of dynamical frequency analysis using adaptive oscillators as basic units.

This is done by constructing a pool of $N$ such oscillators, as illustrated in Fig. 5. The driving signal $F$ is equal to the input (teaching) signal $P_{\text {teach }}$ minus the learned output of the pool $Q_{\text {learned }}$. The properties of the individual adaptive oscillators, i.e. adaptation to frequencies and amplitudes as well as dynamic tracking, are important and exploited by the pool to accomplish dynamical frequency analysis.

Using the negative feedback, the combined output of the oscillators is subtracted from the teaching signal, thus the remaining oscillators only 'feel' the frequency components not fully covered yet by the already adapted oscillators. This is shown experimentally in Fig. 6. When the system starts with uniformly or randomly distributed initial frequencies, the different oscillators will gradually populate the frequency spectrum of the teaching signal. Therefore, frequency analysis is achieved in a fully dynamic way.

Please note that the proposed approach differs from Programmable Central Pattern Generators [10] which have a coupling term between the first oscillator and every other oscillator, to maintain synchronization (constant phase off-
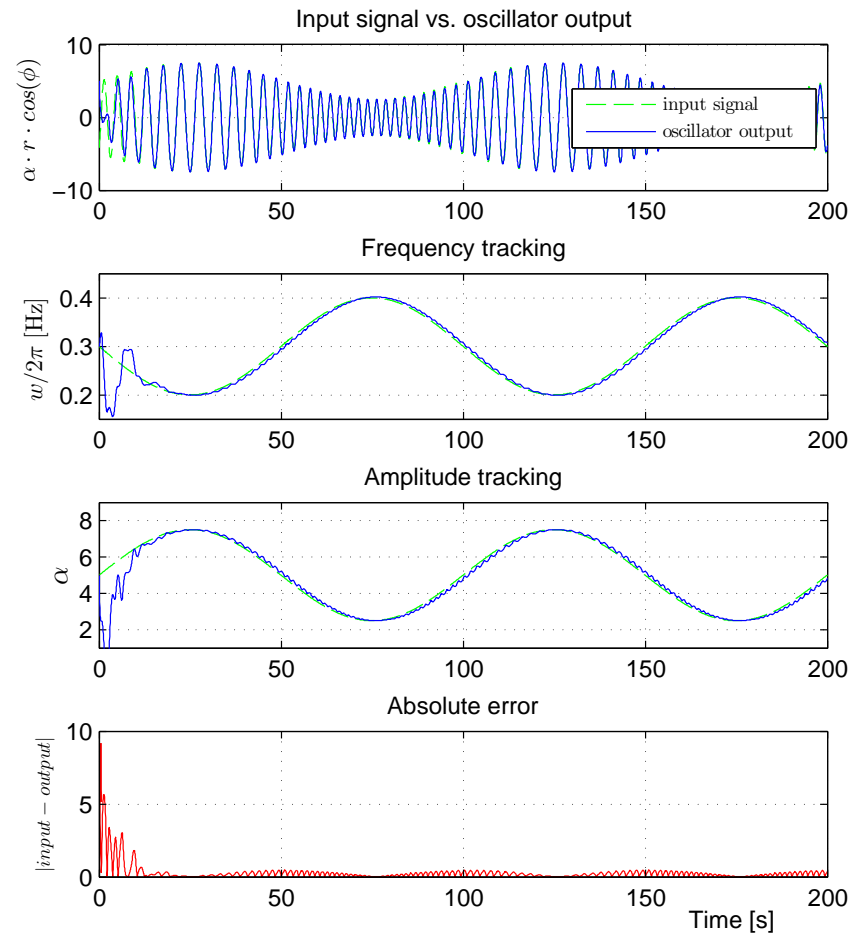

Fig. 4. An adaptive oscillator tracking a non-stationary input signal whose frequency and amplitude both vary over time.

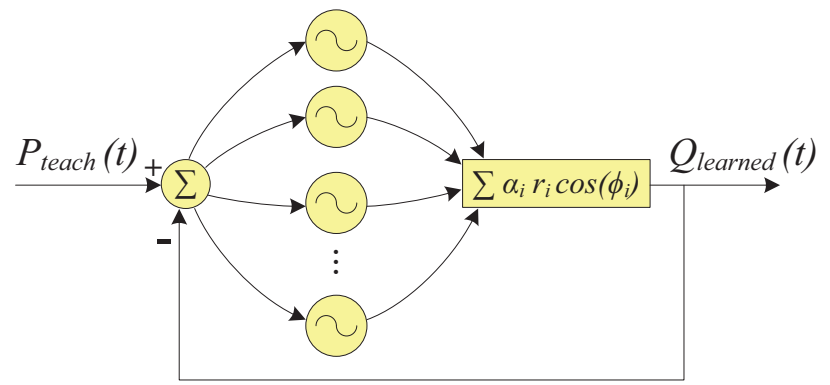

Fig. 5. Pool of multiple adaptive oscillators, as in [8]. Please note that there is no additional coupling between the oscillators other than the mean field negative feedback. This makes the oscillators independent from each other and free to track individual $G_{i}$ components of the input signal.

set). In our case, we do not have such coupling, and each oscillator is free to track its own target frequency independent of the other oscillators.

Another important point is that in the proposed approach there is no possibility for an autonomous mode. The reason for this is that the input signal is never disconnected from the system, so it is constantly in 'learning' mode and never in standalone reproduction/playback mode.

One optional preliminary step that could be added is, instead of uniform random initialization of the frequencies of the oscillators in the pool, to use (one time only) Fourier transform first (e.g. FFT), and then distribute the oscillator frequencies according to the spectrum returned by the Fourier transform. This could speed up the process of initial adaptation to the input signal. 

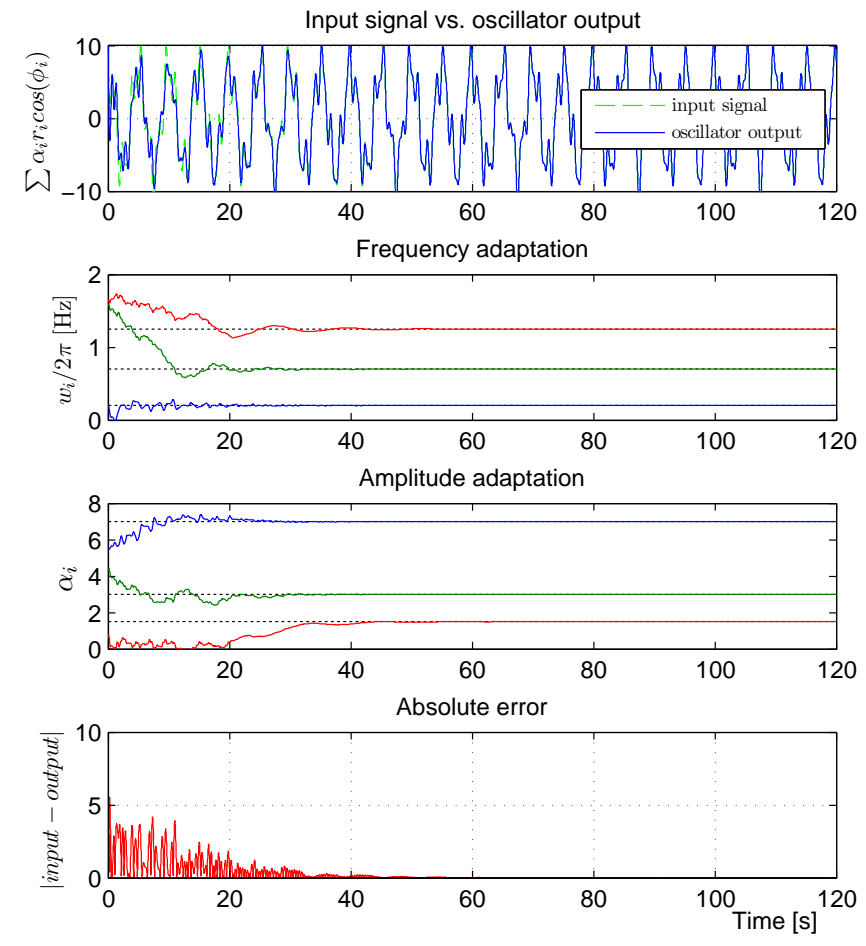

Fig. 6. A pool of 3 oscillators tracking a complex 3-wave input.

\section{INTEGRATION WITH THE AUV CONTROLLER}

To be more specific in the implementation, we assume that the AUV controller is a PID position controller. This, however, can easily be changed to a velocity controller or other type of feedback controller that relies on an error signal.

\section{A. Measuring the external disturbance}

Unfortunately, the external disturbance $F_{\text {ext }}(t)$ cannot be sensed directly or measured explicitly by the AUV. Instead, we need to rely on indirect evidence such as the estimated positional error.

The reason for choosing the positional error as the input/driving signal for learning are two-fold: (i) the error signal includes the combined effect of both the external disturbance $F_{\text {ext }}(t)$ AND the reference trajectory $x_{r e f}(t)$; (ii) the error signal is the input to the PID controller (assuming position control) of the AUV, which allows the proposed approach to intercept it and try to reduce it. Therefore, instead of applying the dynamical frequency analysis on $F_{\text {ext }}(t)$, we apply it on the positional error $e(t)$. The proposed architecture for integrating the developed dynamical frequency analysis part with the AUV controller is illustrated in Fig. 7. In this architecture, the learning part takes as input the positional error, and the output feeds into the AUV controller. An alternative architecture is also possible, where the learning part is at the output of the AUV controller, as explained in [13].

\section{B. Reduction constant}

The reduced error is passed to the PID controller, which in turn produces a reduced control signal, thus saving energy. Figuratively speaking, the approach is 'lying' the PID controller about the actual positional error. The reduced positional error is defined as:

$e_{r}(t)=e(t)-K_{r} \sum_{i=1}^{N} G_{i}(t)=e(t)-K_{r} \sum_{i=1}^{N} \alpha_{i} r_{i} \cos \left(\phi_{i}(t)\right)$,

where $e(t)$ is the actual real positional error, and $e_{r}(t)$ is the reduced error reported to the AUV controller.

The output of the oscillator pool is multiplied by a reduction constant $K_{r} \in[0,1]$ that allows to control the amount of error reduction done by the system. This makes it possible to regulate smoothly the trade-off between energy efficiency and micro-scale tracking precision. For additional safety, it is possible to set a hard-coded upper limit to the error reduction amount, so as to guarantee some prescribed level of tracking precision at all times.

Instead of using numeric differentiation, which is prone to noise, it is possible to use analytic derivative for the $G_{i}$ terms as follows:

$$
\begin{aligned}
& \dot{e}_{r}(t)=\dot{e}(t)-K_{r} \sum_{i=1}^{N} \dot{G}_{i}(t)= \\
& =\dot{x}_{r e f}-\dot{x}_{a c t}+K_{r} \sum_{i=1}^{N} \alpha_{i} r_{i} \sin \left(\phi_{i}(t)\right) .
\end{aligned}
$$

This makes the D-term of the PID controller more stable. In addition, it can be used to calculate a feed-forward term which has low level of noise and more accurately predicts the future value of the error signal.

\section{Absorbing oscillator}

One important addition to the proposed architecture (with respect to Fig. 5) is the additional $\left(N+1^{s t}\right)$ oscillator that we call an absorbing oscillator. Its purpose is to act as a buffer for sudden large disturbances that appear in the input/driving signal. This is achieved by setting the $\epsilon, \eta$ and $\gamma$ parameters of the absorbing oscillator to different values than the ones used for the pool oscillators. In particular, setting $\epsilon$ and $\eta$ to much higher values increases the reactiveness to sudden changes in the input signal, such as sudden peaks, possibly caused by discontinuities in the reference trajectory (e.g. step response) or the environment (e.g. sudden collision). Furthermore, setting $\gamma$ to a smaller value weakens the strength of the attracting limit cycle and helps to absorb quickly any sudden spikes in the signal. Overall, the addition of the absorbing oscillator guarantees that there are no sudden jumps in the output of the oscillator pool, and acts as buffering allowing the oscillator pool enough time to smoothly adapt to changes in the input signal. The importance of this absorbing oscillator is demonstrated by the experimental results in Fig. 11 on a step-response reference trajectory. 


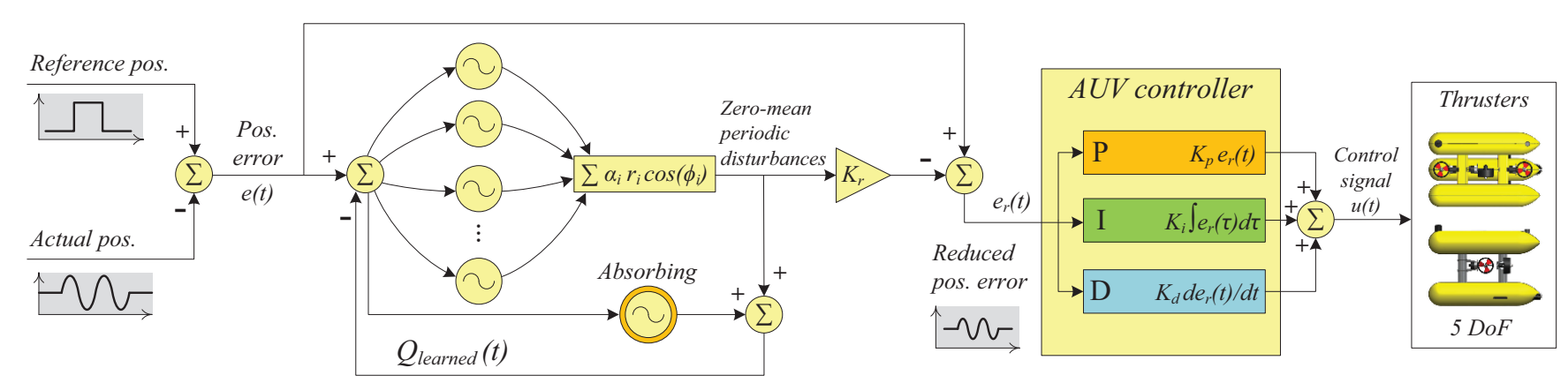

Fig. 7. Integration of the proposed approach with the AUV controller.

\section{EXPERIMENTAL EVALUATION}

For the experimental evaluation we used the specialized UnderWater SIMulator ${ }^{2}$ (UWSim). A screenshot from the simulator is shown in Fig. 8.

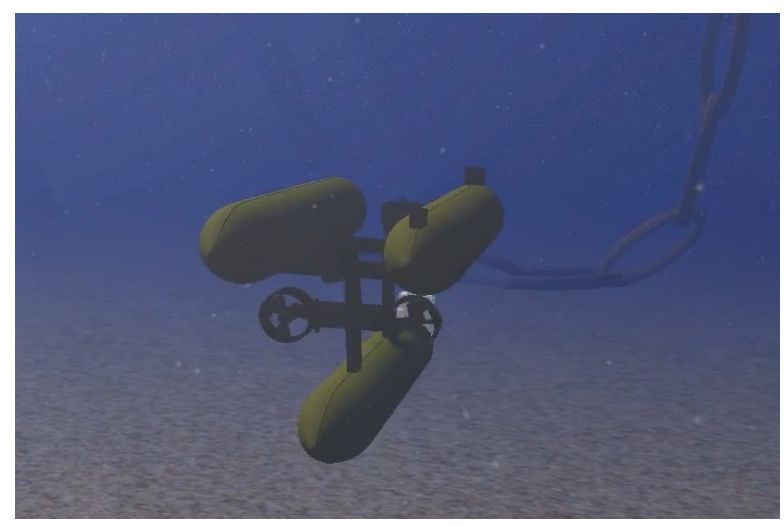

Fig. 8. Screenshot from the UWSim simulation.

\section{A. AUV dynamics model}

This section gives a brief overview of the AUV kinematic and dynamic model used in the experimental evaluation. We consider an underwater vehicle modeled as a rigid body and subject to external forces and torques. According to the standard underwater vehicle modeling properties [14], the dynamic model equations in matrix-vector form are as follows:

$$
\left\{\begin{array}{l}
M \dot{\mathbf{v}}+C(\mathbf{v}) \mathbf{v}+D(\mathbf{v}) \mathbf{v}+g(\eta)=\tau \\
\dot{\eta}=J(\eta) \mathbf{v}
\end{array}\right.
$$

where:

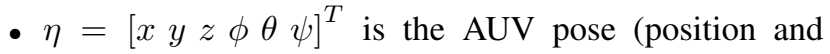
orientation) vector;

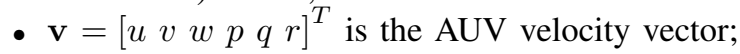

- $M$ is the AUV rigid body inertia matrix;

- $C(\mathbf{v})$ is the rigid body Coriolis and centripetal matrix;

- $D(\mathbf{v})=D_{\text {quad }}(\mathbf{v})+D_{\text {lin }}(\mathbf{v})$ is the quadratic and linear drag matrix respectively;

${ }^{2}$ UWSim - an UnderWater SIMulator for marine robotics research and development, http://www.irs.uji.es/uwsim/.
TABLE I

GIRONA 500 AUV SPECIFICATION

\begin{tabular}{|l|c|}
\hline Weight in air & $140 \mathrm{~kg}$ \\
\hline Length & $1.5 \mathrm{~m}$ \\
\hline Maximum depth & $500 \mathrm{~m}$ \\
\hline Energy & $2.2 \mathrm{kWh} \mathrm{Li-Ion} \mathrm{battery}$ \\
\hline Endurance & $\begin{array}{r}\sim 8 \text { hrs (depending } \\
\text { on speed, payload, etc.) }\end{array}$ \\
\hline $\begin{array}{l}\text { Propulsion system } \\
\text { configuration }\end{array}$ & $\begin{array}{c}5 \text { thrusters }(2 \text { for surge, } \\
2 \text { for heave, } 1 \text { for sway) }\end{array}$ \\
\hline Maximum speed & $1 \mathrm{~m} / \mathrm{s}(2 \mathrm{knots})$ \\
\hline Max. thruster force & $137 \mathrm{~N}(14 \mathrm{kgf})$ \\
\hline Thruster nominal power & $300 \mathrm{~W}$ \\
\hline
\end{tabular}

- $g(\eta)$ is the hydrostatic restoring force vector;

- $J(\eta)$ is the Jacobian matrix transforming the velocities from the body-fixed to Earth-fixed frame;

- $\tau$ is the input (force/torque) vector.

In the experiments we use a dynamics model whose parameters were previously estimated for the Girona 500 AUV using online system identification [15]. The technical specification of Girona 500 is listed in Table I.

\section{B. Wave simulation model}

For modeling the water motion due to surface waves we use the following equations for deep-water waves [16]:

$$
\left\{\begin{array}{l}
\dot{x}(t)=\frac{k g a}{w} \cdot \frac{\cosh (k z(t)+k d)}{\cosh (k d)} \cdot \cos (k x(t)-w t) \\
\dot{z}(t)=\frac{k g a}{w} \cdot \frac{\sinh (k z(t)+k d)}{\cosh (k d)} \cdot \sin (k x(t)-w t),
\end{array}\right.
$$

where $x(t)$ and $z(t)$ are the coordinates of the water particle in the vertical plane containing the wave propagation direction, $d$ is the seafloor depth, $w$ and $a$ are the frequency and amplitude of the wave, respectively, $k$ is the wavenumber (in radians per meter), and $g$ is the gravity constant. The resulting velocity vector field of the water particles is illustrated in Fig. 9.

\section{Experimental results}

The experimental evaluation was conducted with the described dynamics model of the Girona 500 AUV and the described wave model in simulation on two important underwater scenarios: 

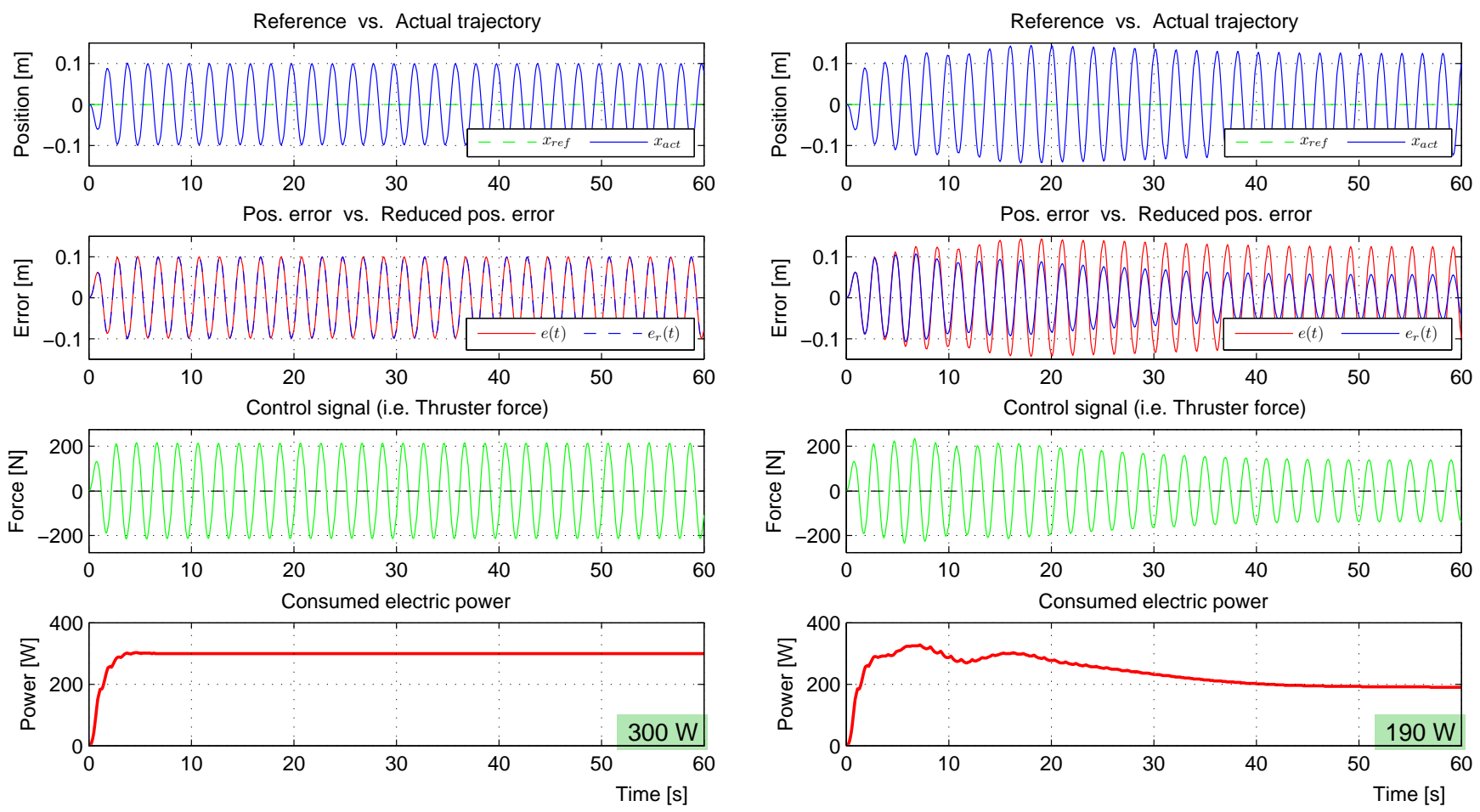

(a) Without the proposed approach $\left(K_{r}=0\right)$

(b) With the approach, $K_{r}=0.5$

Fig. 10. Hovering simulation - hovering at a fixed position while being perturbed by a simulated wave. In (a), the standard PID controller only (without using the proposed approach) consumes around $300 \mathrm{~W}$ electric power during the hovering. In (b), using the same controller and gains, but with the addition of the proposed approach reduces the consumption to only $190 \mathrm{~W}$, which means around $36 \%$ energy saving.
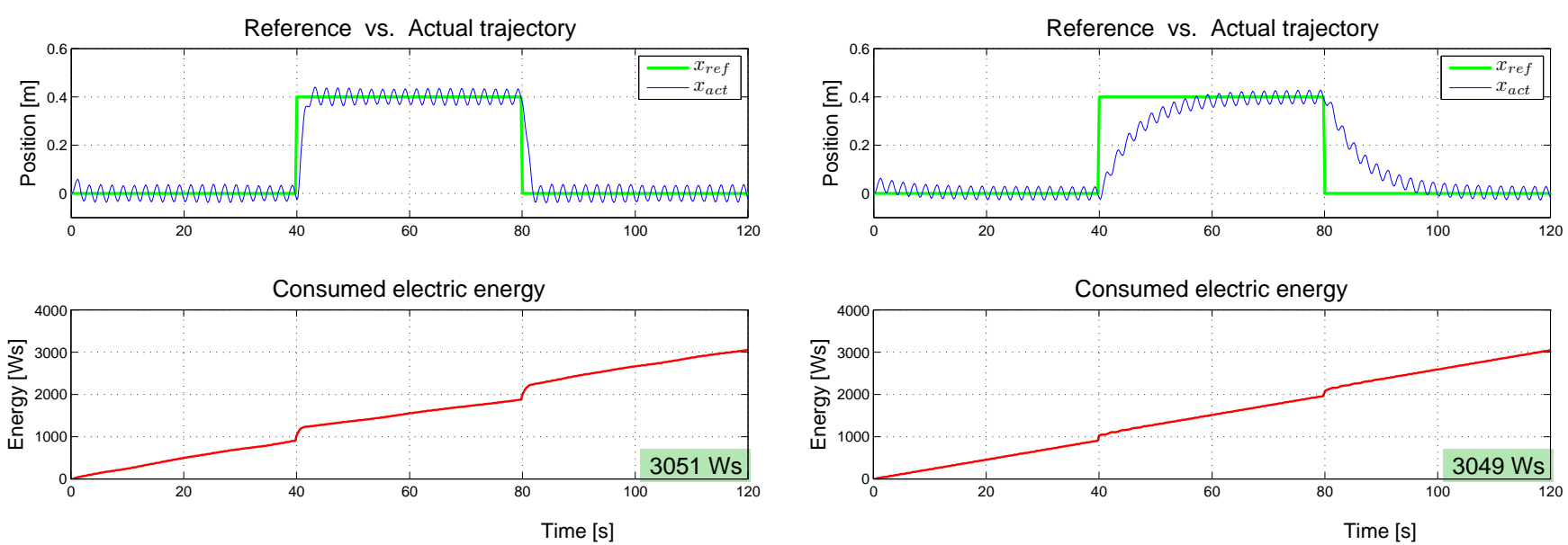

(a) Using the proposed approach, with $K_{r}=0.5$

(b) Without using the approach but with low PID gains.

Fig. 11. Trajectory tracking simulation - tracking a step-response reference trajectory while being perturbed by a simulated wave. In (b) we try to reduce the gains of the standard PID controller in order to match the low energy consumption achieved by the proposed approach in (a) which uses high gains. The results show that an equally-consuming standard controller (b) has much worse tracking performance than the proposed approach (a). This is due to the fact that the proposed approach can use high gains without diminishing the energy-saving capabilities. Thus, for the same amount of consumed energy, the proposed approach achieves much better reference trajectory tracking than a standard controller with low PID gains.

- Hovering simulation - the results are shown in Fig. 10. It shows the significant energy-saving capabilities of the proposed approach.

- Trajectory tracking simulation - the results are shown in Fig. 11. It shows the better tracking performance of the proposed approach compared to an equally-consuming standard controller for the same task.

\section{DISCUSSION}

The proposed approach has numerous advantages that make it suitable for solving the posed problem:

- It is dynamically consistent, and therefore is able to track smoothly the time evolution of non-stationary spectrum;

- It is computationally cheap, and can easily be run inside 


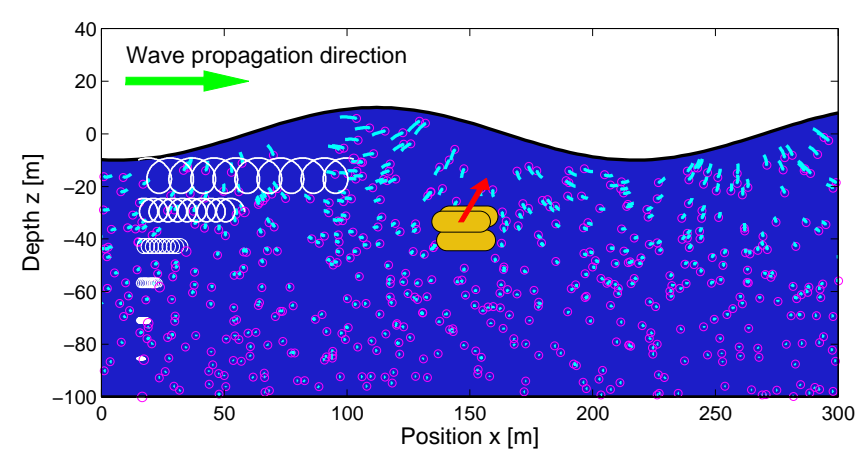

Fig. 9. Wave simulation: showing the estimated velocity vector field of massless particles. The traces of the particles are shown for a fixed duration. The force acting on the AUV is shown with an arrow. This figure is only for illustration purpose - the size of the AUV is not to scale.

the main loop of the AUV controller with negligible impact on the CPU energy consumption;

- It makes accurate predictions of the future input signal. This makes it a good candidate also for the feed-forward terms of more advanced AUV controllers.

The proposed approach is generic in the sense that it can be applied not only to PID position control, but also to other feedback controllers that react to errors in the controlled variables - no matter if it is position, velocity, orientation, or another state variable.

A possible criticism of the proposed approach is that the AUV could simply dive deeper underwater, to avoid the effect of waves. While this is certainly true, there are many reasons why an AUV would swim close to the surface, such as:

- to get a GPS lock in order to update the location estimate and correct for any accumulated dead-reckoning errors;

- to use WiFi or satellite communication to transfer data back to the control center, or ask for assistance and mission update;

- to record sensory data about pollutants in the water at certain shallow depths;

- to perform offshore inspection of a ship hull or other subsea structure (e.g. high risers);

- to perform inspection and cleaning of moving subsea infrastructure such as anchor chains of FPSO ships, which also move periodically due to the surface waves.

Testing the proposed approach in open water with a real AUV would require significant financial, logistic, and human resources, which we, unfortunately, cannot afford at this time. However, our future goal is to establish the necessary collaborations in order to conduct such real-world evaluation of the proposed framework.

\section{CONCLUSIONS}

The original contributions of this paper include:

- novel concept for energy saving of underwater vehicles;

- introduction of synchronization methods in the field of marine robotics;
- first use of adaptive oscillators to learn which disturbances can be ignored;

- addition of an absorbing oscillator (with different parameters than the pool oscillators) for buffering sudden large disturbances;

- mechanism for integration of a PID position controller with dynamical frequency analysis based on adaptive oscillators.

\section{ACKNOWLEDGMENTS}

We would like to thank Arnau Carrera, Narcís Palomeras, and Marc Carreras from the Computer Vision and Robotics Group (VICOROB), University of Girona, Spain, for providing the specialized underwater simulator UWSim and detailed specification of the Girona 500 AUV.

\section{REFERENCES}

[1] D. M. Lane, F. Maurelli, P. Kormushev, M. Carreras, M. Fox, and K. Kyriakopoulos, "Persistent autonomy: the challenges of the PANDORA project," Proceedings of IFAC MCMC, September 2012.

[2] S. R. Ahmadzadeh, P. Kormushev, and D. G. Caldwell, "Autonomous robotic valve turning: A hierarchical learning approach," in Proc. IEEE/RAS Intl Conf. on Robotics and Automation (ICRA), Karlsruhe, Germany, May 2013.

[3] J. G. Graver, "Underwater gliders: Dynamics, control and design," Ph.D. dissertation, Citeseer, 2005.

[4] J. Manley and S. Willcox, "The wave glider: A persistent platform for ocean science," in OCEANS 2010 IEEE-Sydney. IEEE, 2010, pp. $1-5$.

[5] A. Pikovsky, M. Rosenblum, and J. Kurths, Synchronization: A universal concept in nonlinear sciences. Cambridge university press, 2003, vol. 12.

[6] J. Buchli and A. J. Ijspeert, "A simple, adaptive locomotion toysystem," in From animals to animats 8 . Proceedings of the eighth international conference on the simulation of adaptive behavior (SAB04), 2004, pp. 153-162.

[7] R. Ronsse, B. Koopman, N. Vitiello, T. Lenzi, S. M. M. De Rossi, J. van den Kieboom, E. van Asseldonk, M. C. Carrozza, H. van der Kooij, and A. J. Ijspeert, "Oscillator-based walking assistance: A model-free approach," in Rehabilitation Robotics (ICORR), 2011 IEEE International Conference on. IEEE, 2011, pp. 1-6.

[8] L. Righetti, J. Buchli, and A. J. Ijspeert, "From dynamic hebbian learning for oscillators to adaptive central pattern generators," in Proceedings of 3rd International Symposium on Adaptive Motion in Animals and Machines-AMAM 2005, 2005, p. 45.

[9] ——, "Dynamic hebbian learning in adaptive frequency oscillators," Physica D: Nonlinear Phenomena, vol. 216, no. 2, pp. 269-281, 2006.

[10] L. Righetti and A. J. Ijspeert, "Programmable central pattern generators: an application to biped locomotion control," in Proc. IEEE Intl Conf. on Robotics and Automation (ICRA), Orlando, Florida, USA, May 2006, pp. 1585-1590.

[11] J. Buchli, L. Righetti, and A. J. Ijspeert, "Frequency analysis with coupled nonlinear oscillators," Physica D: Nonlinear Phenomena, vol. 237, no. 13, pp. 1705-1718, 2008.

[12] F. Hackenberger, "Balancing central pattern generator based humanoid robot gait using reinforcement learning," Master's thesis, Graz University of Technology, 2007.

[13] P. Kormushev and D. G. Caldwell, "Towards improved AUV control through learning of periodic signals," in Proc. OCEANS 2013 MTS/IEEE, San Diego, USA, September 2013.

[14] T. Fossen, "Guidance and control of ocean vehicles," Wiley, New York, 1994.

[15] G. Karras, C. Bechlioulis, M. Leonetti, N. Palomeras, P. Kormushev, K. Kyriakopoulos, and D. G. Caldwell, "On-line identification of autonomous underwater vehicles through global derivative-free optimization," in Proc. IEEE/RSJ Intl Conf. on Intelligent Robots and Systems (IROS), Tokyo, Japan, November 2013.

[16] R. G. Dean and R. A. Dalrymple, "Water wave mechanics for scientists and engineers," Advanced Series on Ocean Engineering, World Scientific, vol. 2, 1991. 